\title{
Classificação da vegetação e implicações na supressão da Floresta Atlântica: proposta de uma política pública
}

\author{
Renata Jimenez de Almeida-Scabbia ${ }^{1 *}$ (D), Eduardo Pereira Cabral Gomes ${ }^{2}$ (D), Sergio Romaniuc \\ Neto $^{3}$ (D), Luci Mendes Mello Bonini ${ }^{4}$ (D), Maria Santina de Castro Morini ${ }^{5}$ (D) \\ 1 Laboratório de Florística e Sustentabilidade, Núcleo de Ciências Ambietais, Universidade de Mogi das Cruzes, Mogi \\ das Cruzes, SP, Brasil. 08780-911 \\ 2 Núcleo de Ecologia, Instituto de Botânica, São Paulo, SP, Brasil.04301-902. \\ 3 Núcleo Curadoria do Herbário, Instituto de Botânica, São Paulo, SP, Brasil.04301-902. \\ 4 Núcleo de Ciências Sociais Aplicada, Universidade de Mogi das Cruzes, Mogi das Cruzes, SP. 08780-911. \\ 5 Laboratório de Miemecologia do Alto Tietê, Núcleo de Ciências Ambietais, Universidade de Mogi das Cruzes, Mogi \\ das Cruzes, SP. 08780-911. \\ *Autor para correspondência: renatascabbia@hotmail.com
}

Recebido em 22 de janeiro de 2020.

Aceito em 04 de abril de 2021.

Publicado em 15 de abril de 2021.

Resumo - Analisamos como a adoção de diferentes critérios de inclusão para fins de amostragem de vegetação de Mata Atlântica afetam a classificação da mesma nas diferentes fases de sucessão ecológica. A Mata Atlântica Brasileira é um dos tipos florestais mais ameaçados em todo o mundo. A supressão da vegetação nos estágios iniciais é legal, enquanto dos estagios tardios é bem restringida, portanto, a classificação é decisiva para a conservação ou não destes remanescentes florestais. Para a análise utilizamos os dados de um gradiente de perturbação borda-interior em um fragmento de Floresta Atlântica no qual as parcelas amostrais foram alocadas a 5, 15 e $45 \mathrm{~m}$ da borda. Diferentes critérios levaram a conclusões bem diferentes sobre a fase sucessional em que a floresta se encontra e, portanto, é imprescindível a adoção de critérios únicos para a amostragem de vegetação de Floresta Atlântica para fins de classificação da vegetação em estágios sucessionais tendo em vista supressão. Propomos estes critérios que incluem o dap mínimo de inclusão e os grupos taxonomicos indicadores.

Palavras-chave: Floresta Secundária. Mata Atlântica. Meio Ambiente. Política Pública. Resolução Conjunta SMA IBAMA-SP n¹/1994.

\section{Classification of vegetation and implications for clear-cutting of the Atlantic Forest: a public policy proposal}

Abstract - We examine how different inclusion sampling criteria affect classification of the seral stages of Brazilian Tropical Atlantic Forest, one the most threatened forests in the world. Clearcutting of initial sucessional stages is authorized while of late stages it is very restricted, therefore, the classification is fundamental for the conservation or not of the forest remants. It was used data of a gradient edge-interior disturbance in a fragment of tIhis forest in which plots were allocated to 5, 15 and $45 \mathrm{~m}$ of the edge. Different criteria can lead to very different conclusions about the stage of forest sucession, so in this sense, it is necessary to determine unique criteria for the legal 
purposes of clasification of forest sucesion serals with aim of clearcutting vegetation. We propose these criteria that include the minimum inclusion $\mathrm{dbh}$ and the taxonomic indicator groups.

Keywords: Atlantic Forest. Environment. Joint Resolution SMA IBAMA-SP n ${ }^{\circ}$ 1/1994. Public Policy. Secondary Forest.

\section{Clasificación de la vegetación e implicaciones para la tala del Bosque Atlántico: una propuesta de política pública}

Resumen - Examinamos cómo los diferentes criterios de inclusión para el muestreo afectan la clasificación de las distintas etapas de sucesion ecologica en el Bosque Atlántico Tropical Brasileño, uno de los bosques más amenazados del mundo. La talla de las etapas sucesionales iniciales de los bosques es legal, mientras que en las últimas etapas es muy restringido, por tanto, la clasificación es fundamental para la conservación o no destos remanentes forestales. Se utilizaron datos de un gradiente de perturbación borde-interior en un fragmento de bosque Atlantico en el que las parcelas han sido ubicadas a 5, 15 y $45 \mathrm{~m}$ del borde. Diferentes criterios llevaram a conclusiones muy diferentes sobre la etapa de sucesión forestal, por lo que en este sentido, es esencial adoptar criterios únicos para el muestreo de la vegetación del Bosque Atlántico con fines clasificacion de etapas sucesionales para supresión legal. Proponemos estos criterios que incluyen el dap mínimo de inclusión y los grupos taxonómicos indicadores.

Palavras-clave: Bosque Atlántico. Bosque secundario. Medio Ambiente. Política Pública. Resolución Conjunta SMA IBAMA-SP $n^{\circ} 1 / 1994$.

\section{Introdução}

A preocupação com a manutenção da Floresta Atlântica no Brasil não é recente. No período colonial já existiam várias portarias do Governo que davam instruções para se evitar irregularidades e abusos no corte de madeiras (Lima 2011). No final do séc. XVIII foi criado o cargo de Juiz Conservador de Mata que tinha como função preservar as florestas para a exploração de madeira por manejo adequado, proteger as árvores do fogo e mantê-las em áreas declivosas, encostas dos morros e margens dos rios (Pereira 1813). Nessa época, com Baltasar da Silva Lisboa, surge a consciência da preservação das florestas no Brasil (Lima 2011). Em 1786, ele já defendia que as ações dos particulares deveriam ser controladas pelo setor público, pois tudo deveria ser regido para o bem comum. Mesmo assim, depois de 200 anos, os dados sobre esse bioma são alarmantes. A Floresta Atlântica abrangia originalmente, uma área equivalente a $1.310 .299 \mathrm{~km}^{2}$, estendendose por 17 estados, do Rio Grande do Norte ao Rio Grande do Sul. Atualmente, somados todos os fragmentos de floresta nativa com mais de três hectares, restam apenas 12,4\% do bioma original, ou $162.273 \mathrm{~km}^{2}$ (SOS Mata Atlântica 2019). É uma das áreas com os mais elevados índices de endemismo de espécies do planeta, porém muito ameaçada e por isso considerada um hotspot mundial (Ribeiro et al. 2011).

Um marco importante para políticas públicas de conservação deste bioma ocorre com a Constituição Federal de 1988 (Brasil 1988) que a eleva à condição de Patrimônio Nacional e, a partir daí, uma série de decretos e resoluções tentam disciplinar a sua ocupação. Em 1990 é editado o Decreto n 99.547 (Brasil 1990) dispondo sobre a vedação de corte e da respectiva exploração 
da vegetação nativa de Floresta Atlântica. Em 1993 é assinado o Decreto nº 750 (Brasil 1993a) que definiu os limites para o uso e conservação da Mata Atlântica, proibindo o corte, a exploração e a supressão de vegetação primária nos estágios avançado e médio de regeneração.

A primeira iniciativa de regulamentação ocorreu em 1993, com a Resolução CONAMA n¹0 (Brasil 1993b) que estabeleceu parâmetros básicos para análise dos estágios de sucessão da Mata Atlântica. Desde então cada estado da Federação adotou critérios de acordo com as suas características regionais.

No estado de São Paulo a definição de vegetação primária e secundária nos estádios inicial, médio e avançado de regeneração foi estabelecida na Resolução Conjunta SMA IBAMA/SP n ${ }^{\circ} 1$ de 17 de fevereiro de 1994 (São Paulo 1994), utilizando descritores para caracterizar os estágios sucessionais, como a composição florística, presença ou ausência de ervas, epífitas e trepadeiras, diâmetro e altura, espécies indicadoras, entre outros.

Na prática a definição do estágio sucessional determinará se a vegetação será ou não suprimida e a extensão desse processo, com consequências no uso da terra e, portanto, também na economia e na política ambiental (Siminski e Fantini 2004; Siminski et al. 2013).

Os processos biológicos da sucessão podem ser facilmente observados em florestas, seja a partir de casos específicos em um gradiente borda-interior ou por clareiras que são reocupadas progressivamente por diferentes grupos ecológicos de espécies, adaptados às novas condições ambientais que são criadas em razão da abertura do dossel e da sucessiva substituição de espécies ao longo do tempo (Chazdon 2014), ou seja, que uma das características universais de todo o ecossistema é a troca contínua a que está submetido (Rosario 2010). Desde a década de 80 se aceita que a sucessão também ocorre como um processo multidirecional, acontecendo em diferentes escalas temporais (Mori 2011).

A subdivisão do processo de sucessão secundária em fases ou estágios sucessionais distintos, mesmo que arbitrários, é um artifício utilizado na busca do entendimento sobre a dinâmica funcional das florestas (Chazdon et al. 2010). No entanto, a grande diversidade de ecossistemas e complexidade estrutural nos trópicos úmidos faz com que a definição de estágios sucessionais seja dificultada, devido à existência de diversos estágios intermediários antes que se construa um ecossistema similar ao original (Chazdon 2014). Informações sobre essas lacunas de conhecimento podem ser fornecidas pelo estudo do gradiente borda-interior, desde que essa borda possua como interface, uma área completamente desprovida de vegetação.

Vários trabalhos abordam o processo de sucessão secundária no Bioma Mata Atlântica, no Estado de São Paulo (Catharino et al. 2006; Durigan et al. 2008; Rosario 2010). Entretanto, a maior parte destes tem como foco a caracterização do processo ecológico da sucessão natural, poucos têm relacionado este processo às exigências legais de caracterização dos estágios de regeneração da floresta, exceto Rosario (2010). A falta de normatização para a amostragem da vegetação, principalmente no que diz respeito ao inventário florestal exigido para esta caracterização, fragiliza e compromete os valores limites das variáveis estabelecidos pela resolução para diferenciar os estágios de regeneração (Siminski e Fantini 2004; Rosario 2010; Siminski et al. 2013). Autores recomendam que os métodos para o procedimento de campo visando o diagnóstico ambiental sejam baseados em critério científico e definidos em legislação (Duarte et al. 2017; Pacheco et al. 2017). 
A adoção de critérios de amostragem diferentes pode levar a diferentes conclusões, quanto ao estágio de regeneração, em um mesmo conjunto de amostras. Assim, no presente estudo procurouse responder à seguinte questão: a classificação sucessional, segundo a legislação, muda conforme o critério de inclusão adotado? É possível propor uma política ambiental para uniformizar um critério de inclusão na amostragem da vegetação a fim de aprimorar o processo de licenciamento e aplicação da legislação para a supressão de vegetação?

\section{Material e Métodos}

\section{Área de Estudo}

A coleta dos dados foi realizada em um remanescente dentro do Parque Estadual das Fontes do Ipiranga (PEFI), localizado no município de São Paulo (São Paulo). O PEFI está situado no planalto paulista e inserido na Bacia Hidrográfica do Alto Tietê, no domínio Atlântico Brasileiro (Fiaschi e Pirani 2009), em áreas de Floresta Ombrófila Densa (Colombo e Joly 2010) possuindo espécies típicas desta e representantes da Floresta Estacional Semidecídua e até do Cerrado (Barros et al. 2002).

O parque é um trecho de área verde em ambiente intensamente urbanizado, formando uma "ilha florestal" de 336 ha, inserida na metrópole paulistana (Fernandes et al. 2002; Gomes et al. 2003). Localiza-se entre os paralelos $23^{\circ} 38^{\prime} 08^{\prime \prime} \mathrm{S}$ e $23^{\circ} 40^{\prime} 18^{\prime \prime} \mathrm{S}$ e os meridianos $46^{\circ} 36^{\prime} 48^{\prime \prime} \mathrm{O}$ e $46^{\circ} 38^{\prime} 00^{\prime \prime} \mathrm{O}$, em uma área total próxima de $498 \mathrm{ha}$, com altitudes que variam de 760 a $830 \mathrm{~m}$ (Gomes e Mantovani 2001). Possui clima temperado Cwb, segundo a classificação de Köppen, com temperatura média anual de $19,1^{\circ} \mathrm{C}$ e precipitação média de $1.540 \mathrm{~mm}$ por ano (Santos e Funari 2002). O solo de maior ocorrência na área é o latossolo vermelho amarelo. Em estudos taxonômicos da flora fanerogâmica foram registradas 129 famílias, 543 gêneros e 1.159 espécies (Barros et al. 2002).

As parcelas foram alocadas em um trecho de floresta nativa no PEFI, delimitado a sudoeste e sudeste pelas ruas pavimentadas internas do Jardim Botânico, ao norte pelo arboreto e a leste por edificações. O remanescente passou por perturbação mais intensa entre o fim do século XIX e início do XX quando parte do material dragado do lago adjacente foi depositado em seu interior. A área é fechada a visitação e está livre de perturbação antrópica direta há pelo menos 70 anos. Para o estudo foi escolhida a borda sudoeste, por apresentar uma condição intermediária de luminosidade e menor variação de altitude ao longo de seus limites (Tanus et al. 2012).

\section{Coleta de dados}

Foram estabelecidos dez transectos da borda para o interior da floresta. Em cada um deles foram instaladas três parcelas circulares de $50 \mathrm{~m}^{2}$ a $5 \mathrm{~m}$ (partindo do centro da circunferência), 15 $\mathrm{m}$ e $45 \mathrm{~m}$ da borda. A distância entre os transectos foi aleatoriamente estabelecida, observando-se a distância mínima de $20 \mathrm{~m}$ entre eles.

Partiu-se do pressuposto que o gradiente borda-interior espacializa um gradiente de perturbação, de mais para menos perturbado, e o efeito da distância é exponencial sendo mais intenso a curtas distâncias (van Dyke 2008), o que foi confirmado pelo trabalho de Tanus et al. 
(2012). A partir daí três critérios para as comparações foram selecionados: i) diâmetro a altura do solo (DAS) para indivíduos com ao menos $1,5 \mathrm{~m}$ de altura; ii) diâmetro a altura do peito (DAP) $>5,0 \mathrm{~cm}$, e; iii) DAP $>10,0 \mathrm{~cm}$.

Todos os indivíduos lenhosos, vivos ou mortos, em pé, com no mínimo 1,5 $\mathrm{m}$ de altura foram incluídos. Cada indivíduo amostrado foi marcado com a fixação de uma placa numerada em seu tronco. Foram realizadas coletas semanais de dezembro de 2013 a maio de 2014. A nomenclatura botânica utilizada para a denominação das famílias seguiu a classificação proposta em APG IV (2016) e para as espécies a Flora do Brasil (2021).

\section{Análise dos dados}

Para cada critério ao longo do gradiente $(5,15$ e $45 \mathrm{~m})$ a riqueza de espécies a diferentes distâncias da borda foi comparada objetivamente por rarefação (Goteie e Graves 1996). Neste método a abundância é controlada, i.e., a comparação é feita fixando-se o número de indivíduos. Assim, foram realizados 1.000 sorteios de 250 indivíduos para o conjunto de parcelas a cada distância, e a partir da média da riqueza e o respectivo intervalo de confiança foi feita a comparação entre as diferentes distâncias.

Para cada critério de inclusão, foi calculada a simetria (Sokal e Rohlf 1995) das curvas de distribuição de diâmetro e de altura do total amostrado a cada distância.

A partir da Resolução Conjunta SMA IBAMA/SP n 1 de 17 de fevereiro de 1994 (São Paulo 1994) foram selecionados parâmetros que poderiam ser afetados por diferentes critérios de inclusão e, consequentemente influenciariam na classificação sucessional da área estudada. Entre estes podem ser citados: densidade, área basal, altura e diâmetro médios, além da diversidade biológica. Diferenças significativas entre estes valores ao longo do gradiente foram avaliados por Análise de Variância de um fator tendo as dez parcelas como réplicas.

\section{Resultados e Discussão}

O número de indivíduos incluídos diminui conforme o tamanho mínimo de inclusão aumenta, o que é esperado. Além disso, as curvas de distribuição de classes de diâmetro e altura, chegam a mudar em alguns casos (Figura 1), apresentando valores de simetria de sinais opostos à medida que menos indivíduos são incluídos. 
Figura 1. Distribuição por classes de diâmetro (DAS=diâmetro a altura do solo e $\mathrm{DAP}=$ diâmetro a altura do peito) e altura, indivíduos (+) e S (assimetria da curva de distribuição), para cada critério de inclusão e distância da borda no Parque Estadual Fontes do Ipiranga, São Paulo (SP).

\section{Critério de inclusão}
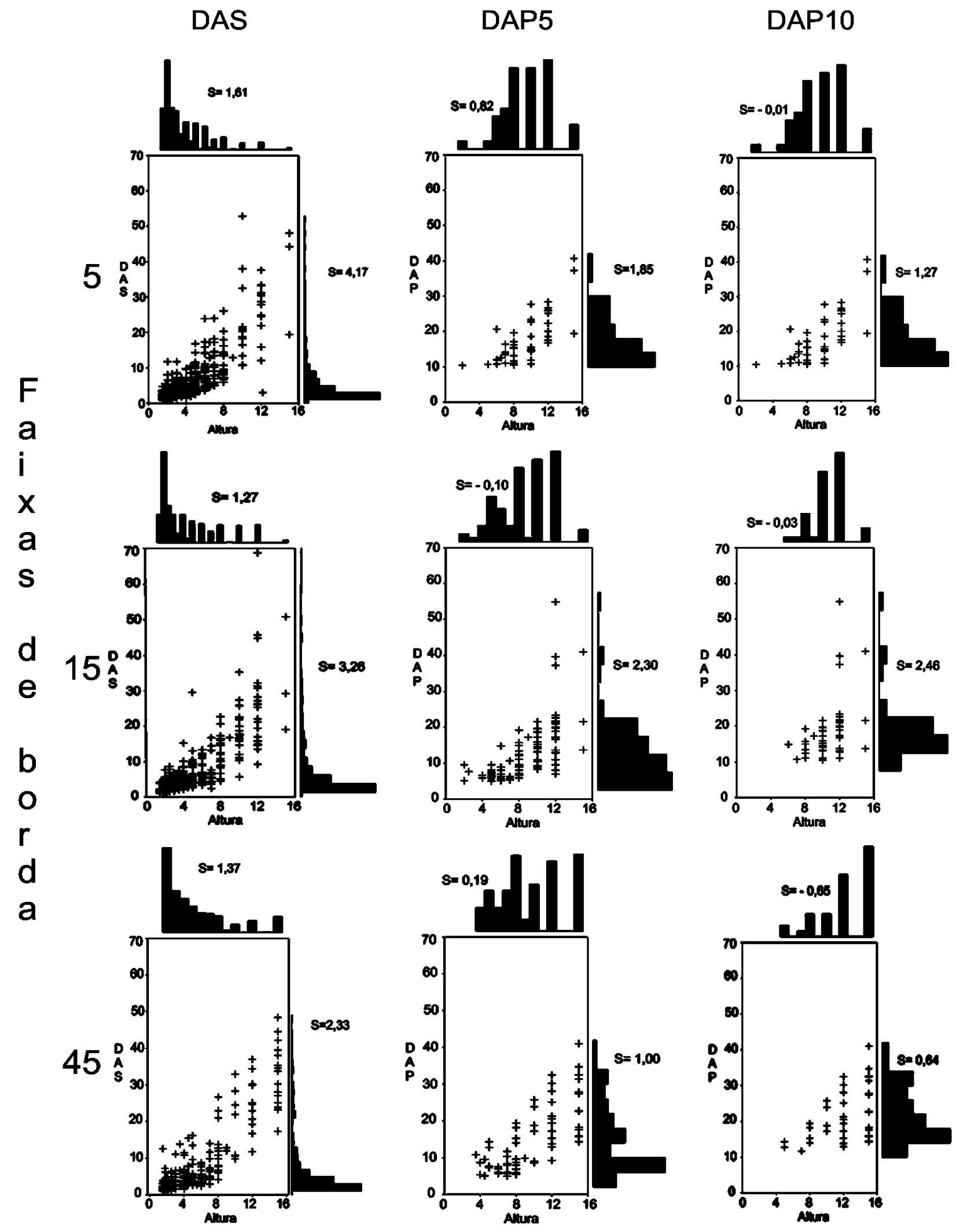
A estimativa de DAP médio, sugerida pela Resolução CONAMA (São Paulo 1994) também mostrou bastante variação entre os critérios (tabela 1). O DAP médio resultante aumentou cerca de três vezes quando o critério passou de 1,5m-DAS para DAP-10. Sob o critério mais inclusivo as parcelas a $5 \mathrm{~m}$ da borda foram significativamente diferentes das parcelas a 15 e $45 \mathrm{~m}$ (ANOVA 1 fator, 2 g.l., $F=26,06 \mathrm{p}<0,001$ ), porém para $\mathrm{DAP} \geq 5 \mathrm{e} \geq 10$ não houve diferenças significativas entre as médias de DAP (ANOVA 1 fator, 2 g.l., $\mathrm{F}=2,90$ e $\mathrm{F}=0,92, \mathrm{p}=0,0723$ e $\mathrm{p}=0,41$ respectivamente).

Verificou-se que para a altura média, o valor mais que dobra do menor para o maior critério mostrando o quanto este valor muda. Para o primeiro critério (1,5-DAS) todas as curvas apresentam simetria positiva, enquanto que para o último (DAP-10), todas negativas.

A riqueza sempre foi máxima na borda, independente do critério de inclusão adotado (Tabela 1). Utilizando-se 1,5-DAS não foi observada diferença significativa (ANOVA 1 fator, 2 g.l., $F=2,71$, $\mathrm{p}=0,08$ ), ao longo do gradiente de perturbação, por outro lado com o critério DAP $\geq 5 \mathrm{~cm}$ e DAP $\geq 10 \mathrm{~cm}$, as parcelas menos perturbadas (a $45 \mathrm{~m}$ da borda) apresentaram riqueza significativamente maior (ANOVA 1 fator, 2 g.l., $F=26,3, p<0,0001$ e $F=17,7, p<0,0001$, respectivamente). Em parte, a diferença nos padrões encontrados deve-se ao maior número de indivíduos amostrados nas proximidades da borda.

Ao controlar-se este fator fazendo-se a comparação por rarefação, isto é, fixando-se o mesmo número de indivíduos para as três distâncias não há diferenças com o critério mais inclusivo. Provavelmente isto se deve a exclusão de arbustos pioneiros heliófitos, principalmente das famílias Asteraceae, Solanaceae e Melastomataceae, de pequeno porte que não são amostrados a partir de um determinado diâmetro.

O critério mais restritivo leva a uma diminuição mais acentuada da riqueza na borda do que no interior o que explica a maior riqueza (fixando-se o número de indivíduos por rarefação) a $45 \mathrm{~m}$.

A partir do parâmetro "Área Basal" a situação das parcelas a $45 \mathrm{~m}$ da borda chega a se inverter: com o critério mais inclusivo apresentam a menor área basal ao longo do gradiente, com DAP $\geq$ $10,0 \mathrm{~cm}$ a maior (Tabela 1 ).

Tabela 1. Caracterização de estrutura em três faixas de borda, em diferentes estágios sucessionais no Parque Estadual Fontes do Ipiranga, São Paulo (SP). Valores entre parênteses representam o estágio sucessional em que o valor pode ser enquadrado de acordo com a Resolução Conjunta SMA IBAMA/ SP $n^{\circ} 1$ de 17 de fevereiro de 1994. Critério = critério de inclusão (DAS = diâmetro a altura do solo; dap

= diâmetro a 1,3 $\mathrm{m}$ do solo; dist = distância em metros da borda; dapMd = dap médio $(\mathrm{cm}) ;$ AltMd = altura média $(\mathrm{m}) ; \mathrm{AB}=$ área basal $(\mathrm{m} 2) ; \mathrm{D}=$ densidade (ind./ha); $\mathrm{S}=$ número de espécies por rarefação (o mesmo número de indivíduos amostrados para todas as parcelas); $\mathrm{R}=$ riqueza total; NspDom = número de espécies dominantes (número de espécies que em ordem decrescente contribuíram ao menos com 50\% do total de indivíduos); Fam = as cinco famílias mais abundantes.

\begin{tabular}{|c|c|c|c|c|c|c|c|c|c|}
\hline Critério & dist & dapMd & AltMd & AB & D & spp & $\mathbf{R}$ & NspDom & Fam \\
\hline & 5 & $5,2(\mathrm{i})^{\mathrm{A}}$ & $3,7(\mathrm{i})^{\mathrm{A}}$ & 79,31 & $13.040^{\mathrm{A}}$ & $80,8 \pm 3,7$ & 112 & 15 & $\begin{array}{l}\text { Euphorbiaceae, } \\
\text { Fabaceae, } \\
\text { Lauraceae, } \\
\text { Melastomataceae, } \\
\text { Rubiaceae }\end{array}$ \\
\hline 1,5 -DAS & 15 & $6,5(\mathrm{i})^{\mathrm{B}}$ & $4,3(\mathrm{i}, \mathrm{m})^{\mathrm{AB}}$ & 85,07 & $8.620^{\mathrm{B}}$ & $82,0 \pm 3,1$ & 100 & 14 & $\begin{array}{l}\text { Euphorbiaceae, } \\
\text { Lauraceae, } \\
\text { Rubiaceae, } \\
\text { Arecaceae, } \\
\text { Myrtaceae }\end{array}$ \\
\hline
\end{tabular}




\begin{tabular}{|c|c|c|c|c|c|c|c|c|c|}
\hline Critério & dist & dapMd & AltMd & $A B$ & D & spp & $\mathbf{R}$ & NspDom & Fam \\
\hline & 45 & $7,4(\mathrm{i})^{\mathrm{B}}$ & $4,9(\mathrm{i}, \mathrm{m})^{\mathrm{B}}$ & 74,84 & $5.900^{\mathrm{B}}$ & $79,9 \pm 0,3$ & 82 & 15 & $\begin{array}{l}\text { Arecaceae, } \\
\text { Rubiaceae, } \\
\text { Euphorbiaceae, } \\
\text { Myrtaceae, } \\
\text { Meliaceae }\end{array}$ \\
\hline \multirow{3}{*}{$\mathrm{DAP} \geq 5,0$} & 5 & $10,7(\mathrm{~m})^{\mathrm{A}}$ & $7,1(\mathrm{i}, \mathrm{m})^{\mathrm{A}}$ & 40,85 & $2.760^{\mathrm{A}}$ & $33,9 \pm 2,2$ & 45 & 7 & $\begin{array}{l}\text { Euphorbiaceae, } \\
\text { Fabaceae, } \\
\text { Melastomataceae, } \\
\text { Lauraceae, } \\
\text { Primulaceae }\end{array}$ \\
\hline & 15 & $12,4(\mathrm{~m})^{\mathrm{A}}$ & $8,2(\mathrm{~m}, \mathrm{a})^{\mathrm{AB}}$ & 44,89 & $2.160^{A B}$ & $34,2 \pm 1,7$ & 40 & 8 & $\begin{array}{l}\text { Euphorbiaceae, } \\
\text { Arecaceae, } \\
\text { Meliaceae, } \\
\text { Myrtaceae, } \\
\text { Lauraceae }\end{array}$ \\
\hline & 45 & $14,1(\mathrm{~m})^{\mathrm{A}}$ & $9,2(\mathrm{~m})^{\mathrm{B}}$ & 43,88 & $1.660^{\mathrm{B}}$ & $38,7 \pm 0,5$ & 40 & 8 & $\begin{array}{l}\text { Arecaceae, } \\
\text { Euphorbiaceae, } \\
\text { Rubiaceae, } \\
\text { Myrtaceae, } \\
\text { Meliaceae }\end{array}$ \\
\hline \multirow{3}{*}{$\mathrm{DAP} \geq 10$} & 5 & $17,6(\mathrm{~m})^{\mathrm{A}}$ & $9,1(\mathrm{~m}, \mathrm{a})^{\mathrm{A}}$ & 33,67 & $1.000^{\mathrm{A}}$ & $21,2 \pm 1,0$ & 25 & 5 & $\begin{array}{l}\text { Euphorbiaceae, } \\
\text { Fabaceae, } \\
\text { Melastomataceae, } \\
\text { Lauraceae, } \\
\text { Asteraceae }\end{array}$ \\
\hline & 15 & $17,7(\mathrm{~m})^{\mathrm{A}}$ & $10,0(a)^{A B}$ & 39,21 & $1.100^{\mathrm{A}}$ & $19,9 \pm 0,8$ & 25 & 6 & $\begin{array}{l}\text { Euphorbiaceae, } \\
\text { Arecaceae, } \\
\text { Myrtaceae, } \\
\text { Lauraceae, } \\
\text { Meliaceae }\end{array}$ \\
\hline & 45 & $20,3(a)^{A}$ & $11,6(a)^{\mathrm{B}}$ & 40,09 & $880^{\mathrm{A}}$ & $21,6 \pm 0,5$ & 24 & 5 & $\begin{array}{l}\text { Arecaceae, } \\
\text { Euphorbiaceae, } \\
\text { Fabaceae, } \\
\text { Annonaceae, } \\
\text { Sapotaceae }\end{array}$ \\
\hline
\end{tabular}

Tais observações tem implicações práticas, pois com a redução de indivíduos amostrados em relação ao aumento no DAP do critério de inclusão, o esforço amostral fica comprometido. Concordando com Siminski e Fantini (2004) e Siminski et al. (2013) os dados sugerem que a inclusão de indivíduos com DAP $<5 \mathrm{~cm}$ implicam, na medição de um número excessivamente grande de plantas. $\mathrm{O}$ oposto acontece quando se medem somente indivíduos com DAP $\geq 10 \mathrm{~cm}$. Desta forma, a medição de indivíduos com DAP $\geq 5 \mathrm{~cm}$ dentro da parcela do inventário mostrase como o critério que permite melhor conciliar a caracterização do estágio sucessional com a operacionalidade nesse tipo de inventário. Além disso, o DAP é medido mais fácil e rapidamente, o que simplifica os levantamentos, além de possibilitar maior precisão e exatidão das estatísticas, quando comparado às estimativas de altura.

Atualmente a única resolução que apresenta um critério definido, é a Resolução CONAMA no. 02/1994 (Paraná 1994), que estabelece os parâmetros para definir os estágios sucessionais no estado 
do Paraná, adotando como critério a circunferência a altura do peito (CAP) igual ou superior a 20 $\mathrm{cm}(6,36 \mathrm{~cm}$ de DAP).

É importante ressaltar que o desenvolvimento de políticas conservacionistas ou de supressão em todo o território nacional e o aproveitamento racional dos recursos oferecidos pela Mata Atlântica, são diretamente afetados pela definição dos estágios sucessionais. Tal definição passa por três problemas: i) o alto grau de especialização necessária para essa caracterização, desde a realização da identificação florística até a análise quantitativa dos dados; ii) a vegetação que muitas vezes apresenta uma grande heterogeneidade e consequentemente, gera iii) a aplicação da Resolução, pois os termos "baixo" ou "significativo" são subjetivos e podem ser compreendidos a partir de diferentes interesses, e muitas vezes possuem características que se sobrepõem (Tabela 1). Um mesmo valor pode ser interpretado de formas diferentes, o que, na prática gera inúmeros conflitos, que acabam se arrastando por anos no judiciário.

Neste estudo, por exemplo, a interpretação de valores quantitativos como a riqueza e a dominância das espécies, mostra que quanto mais inclusivo, maior o número total de espécies e, entre essas, as espécies dominantes. Considerando-se que os maiores valores apresentados são as maiores riquezas, tem-se uma completa inversão de resultados (Tabela 1).

Em relação aos estratos dominantes para estágio inicial e médio (São Paulo 1994), o significado de "estratos lenhosos variando de abertos a fechados apresentando plantas com alturas variáveis" e a "presença de camadas de diferentes alturas, sendo que cada camada apresenta-se com cobertura variando de aberta a fechada", respectivamente, não apresentam diferença na prática. No caso do estágio médio "podendo (...) aparecer árvores emergentes" não facilita a interpretação.

Existe também a questão da identificação das espécies vegetais características, o que demanda uma alta especialização técnica. Além disso, tal identificação pode ser questionada, se o material utilizado não possuir estruturas reprodutivas. Nos levantamentos o que mais se observa são materiais desprovidos de tais estruturas, principalmente com critérios mais inclusivos. Diante disso a utilização de famílias como indicadoras, em conjunto com o parâmetro da área basal seriam mais indicados, pois se sabe que algumas famílias são características de determinados estágios, por exemplo Asteraceae, Melastomataceae e Solanaceae para estágio inicial e Myrtaceae para estágio avançado (Günter et al. 2007; Gomes et al. 2020), o que já é adotado pela legislação.

Concordando com Duarte et al. (2017) metodologias para coleta de dados no campo, para fins de diagnóstico, devem ser estabelecidos em legislação, com bases científicas. Tornando os processos de licenciamento mais transparentes.

\section{Conclusão}

Esta estudo tinha como objetivo mostrar que a adoção de critérios de amostragem diferentes pode levar a diferentes conclusões, quanto ao estágio de regeneração, em um mesmo conjunto de amostras. Entende-se que esses objetivos foram atingidos pois os resultados mostram que diferentes critérios ordenam e classificam os mesmos trechos de vegetação amostrados de maneira diferente. Conforme o critério, a sequência de ordenação, ou seja, as curvas de distribuição de classes de diâmetro e altura, chega a ser oposta. 
Diante disso, propõe-se uma revisão das normas legais de supressão que balizam as políticas públicas atuais para que se possa inserir um critério de inclusão, como demonstrado neste estudo de caso: o diâmetro a altura do peito $(\mathrm{DAP}) \geq 5,0 \mathrm{~cm}$, mostrou-se o mais adequado.

Também, entendeu-se que, nesta norma, deve ser observada a área da amostragem em um mesmo fragmento pois dependendo de onde ela for instaladacomo, pode apresentar características iniciais, como foi o caso da faixa de $5 \mathrm{~m}$ na borda, devido às condições ambientais às quais ela está exposta, jamais sairá, do estágio inicial, já o interior do fragmento, ou seja a $15 \mathrm{~m}$, a vegetação apresenta características de estágio avançado, logo seria importante adotar esse critério para fins legais de supressão e/ou conservação.

Finalmente, propõe-se também que a legislação contemple o critério de adoção de famílias botânicas para determinação de estágio sucessional, uma vez que sua identificação exige menor qualificação técnica e assim menor probabilidade de erros. A junção de área basal e famílias melhoraria a caracterização fitofisionômica, pois a utilização de espécies como parâmetro, também gera uma grande quantidade de incertezas, pois a literatura que permite identificação a partir de material estéril é escassa, especialmente para espécies raras ou em listas vermelhas.

Outros estudos podem contemplar novas áreas usando-se a mesma metodologia de modo que se possa configurar a validade do que aqui está exposto.

\section{Agradecimentos}

A Capes pela bolsa de pós doutorado concedida. Ao Instituto de Botânica de São Paulo e a Universidade de Mogi das Cruzes (UMC). Aos revisores, pelas contribuições.

Participação dos autores: RJAS, EPCG, SRN - coleta de campo, triagem de material, análise dos dados; LMMB, MSCM - revisão do manuscrito, coordenação do projeto.

Aprovação ética e outras licenças: não se aplica.

Disponibilidade dos dados: Os dados não estarão disponíveis em base ou repositores.

Fomento: concessão de bolsa de pós-doutorado pela Coordenação de Aperfeiçoamento de Pessoal de Nível Superior (Capes) à primeira autora.

Conflito de Interesses: os autores declaram não haver conflito de interesses.

\section{Referências}

APG IV (Angiosperm Phylogeny Group). 2016. An update of the Aniosperm Phylogeny Group classification for the orders and families of flowering plants: APG IV. Botanical Journal of the Linnean Society, 181:105-121. DOI: 10.1111/ boj. 12385

Barros F. 2002. A flora fanerogâmica do PEFI: composição, afinidades e conservação. In: Bicudo DC et al. (orgs.), Parque Estadual das Fontes do Ipiranga (PEFI): unidade de conservação que resiste à urbanização de São Paulo. São Paulo: Secretaria do Meio Ambiente do Estado de São Paulo, Brasil, p. 93-110.

Brasil. 1988. Constituição da República Federativa do Brasil. Brasília, DF: Senado Federal: Centro Gráfico, 292 p.

Brasil. 1990. Decreto n. 99.547, de 25 de setembro de 1990. Diário Oficial da União, Brasília, DF (1990 set. 28); Sec. 1: 18463. 
Brasil. 1993a. Decreto n. 750, de 10 de fevereiro de 1993. Diário Oficial da União, Brasília, DF (1993fev. 11); 1801.

Brasil. 1993b. CONAMA. Resolução n. 10 de 1. de outubro de 1993. Diário Oficial da União, Brasília, DF (1993 nov. 03); 16497-16498.

Catharino ELM, Bernacci LC, Franco GADC, Durigan G, Metzger JP. 2006. Aspectos da composição e diversidade do componente arbóreo das florestas da Reserva Florestal do Morro Grande, Cotia, SP. Biota Neotropica, 6(2):1-22. DOI: $10.1590 /$ S1676-06032006000200004

Chazdon RL. 2014. Second growth: The promise of tropical forest regeneration in an age of deforestation. Chicago: University of Chicago Press, USA, $472 \mathrm{p}$.

Chazdon RL, Finegan B, Capers RS, Salgado-Negret B, Casanoves F, Boukili V, Norden N. 2010. Composition and dynamics of functional groups of trees during tropical forest succession in northeastern Costa Rica. Biotropica, 42(1):3140. DOI: $10.1111 / \mathrm{j} .1744-7429.2009 .00566 . x$

Colombo AF, Joly CA. 2010. Brazilian Atlantic Forest lato sensu: the most ancient Brazilian forest, and a biodiversity hotspot, is highly threatened by climate change. Brazilian Journal of Biology, 70(3):697-708. DOI: 10.1590/S151969842010000400002

Duarte CG, Dibo APA; Sánchez LE. 2017. O que diz a pesquisa acadêmica sobre avaliação de impacto e licenciamento ambiental no Brasil. Ambiente \& Sociedade, 20(1):245-278. DOI: 10.1590/1809-4422asoc20150268r1v2012017.

Durigan G, Bernacci LC, Franco GADC, Arbocz GF, Metzger JP, Catharino ELM. 2008. Estádio sucessional e fatores geográficos como determinantes da similaridade florística entre comunidades florestais no Planalto Atlântico, Estado de São Paulo, Brasil. Acta Botanica Brasilica, 22(1):51-62. DOI: 10.1590/S0102-33062008000100007

Fernandes AJ, dos Reis LAM, Carvalho A. 2002. Caracterização do meio físico. In: Bicudo DC et al. (orgs.). Parque Estadual das Fontes do Ipiranga: unidade de conservação que resiste à urbanização de São Paulo. São Paulo: Secretaria do Meio Ambiente de São Paulo, Brasil, p. 51-62.

Fiaschi P, Pirani, JR. 2009. Review of plant biogeographic studies in Brazil. Journal of Systematics and Evolution, n. 47, p. 477-496. DOI: $10.1111 / j .1759-6831.2009 .00046 . x$

Flora do Brasil. 2021. Flora do Brasil 2020 em construção. Jardim Botânico do Rio de Janeiro. Disponível em: < http:// floradobrasil.jbrj.gov.br/reflora/listaBrasil/ConsultaPublicaUC/ConsultaPublicaUC.do\#CondicaoTaxonCP / >. Acesso em: 05 abr. 2021.

Gomes EPC, Mantovani W. 2001. Size structure in a warm temperate forest tree populations in São Paulo, SP, Southeastern Brazil. Naturalia, 26:131-158.

Gomes EPC, Mantovani W, Kageyama PY. 2003. Mortality and recruitment of trees in a secondary montane rain forest in Southeastern Brazil. Brazilian Journal of Biology, 63:47-60.

Gomes EPC, Sugiyama M, Oliveira Junior CJF, Prado HM, Ribeiro Filho AA, Adams C. 2020. Post-agricultural succession in the fallow swiddens of Southeastern Brazil. Forest Ecology and Management, 475: 118398. DOI: 10.1016/j. foreco.2020.118398

Gotelli NJ. and Graves GR. 1996.Null Models in Ecology. Washington, DC: Smithsonian Institution Press.

Günter S, Weber M, Erreis R, Aguirre N. 2007. Influence of distance to forest edges on natural regeneration of abandoned pastures: a case study in the tropical mountain rain forest of Southern Ecuador. European Journal of Forest Research, 126:67-75. DOI: 10.1007/s10342-006-0156-0

Lima APS. 2011. Baltasar da Silva Lisboa: O Juiz Conservador das Matas de Ilhéus (1797-1818). Revista Crítica Histórica, 4:160-175. Disponível em <http://www.seer.ufal.br/index.php/criticahistorica/article/view/2771/pdf>. Acesso em: 05 jan. 2021. 
Mori AS. 2011. Ecosystem management based on natural disturbances: hierarchical context and non-equilibrium paradigm. Journal of Applied Ecology, 48(2):280-292. DOI: 10.1111/j.1365-2664.2010.01956.x

Pacheco LM, Dantas MK, Passador CS, Liboni-Amui LB. 2017. Environmental public expenses: an integrative literature review and future research agenda. Ambiente \& Sociedade, 20(4):209-228. DOI: 10.1590/1809-4422asoc0138r2v2042017

Paraná. 1994. Resolução CONAMA n. 2 de 18 de março de 1994. DOU n. 59, de 28 de março de 1994, Seção 1, p 4513-4514. Disponível em: < http://snif.florestal.gov.br/images/pdf/legislacao/resolucoes_conselho/resolucao_conama_02_1994. pdf>. Acesso em: 05abr. 2021.

Pereira JMD. 1813. Elogio Historico do Senhor D. Pedro Carlos de Bourbon e Bragança, Infante de Hespanha, e Portugal: Almirante General da Marinha Portugueza: composto e oferecido a' muito augusta Princeza a senhora Dona Maria Thereza viúva do mesmo senhor. Rio de Janeiro: Impressão Régia, 70 p. Disponível em: <https://digital.bbm.usp.br/ handle/bbm/7509> . Acesso em: 05 abr. 2021.

Ribeiro MC, Martensen AC, Metzger JP, Tabarelli M, Scarano FR, Fortin MJ. 2011. The Brazilian Atlantic Forest: a shrinking biodiversity hotspot. In: Zachos FE and Habel JC (org.). Biodiversity Hotspots: Distribution and Protection of Conservation Priority Areas. Berlin-Heidelberg: Springer, p. 405-434.

Rosario RPG. 2010. Estágios sucessionais e o enquadramento jurídico das florestas montanas secundárias na Reserva Florestal do Morro Grande (Cotia, SP) e entorno. Dissertação (Mestrado em Biodiversidade Vegetal e Meio Ambiente) - Instituto de Botânica da Secretaria de Estado do Meio Ambiente, São Paulo, $153 f$.

Santos PM and Funari FL. 2002. Clima local. In: Bicudo DC et al.; (orgs.). Parque Estadual das Fontes do Ipiranga: unidade de conservação que resiste à urbanização de São Paulo. São Paulo: Secretaria do Meio Ambiente do Estado de São Paulo, p. 27-46.

São Paulo. 1994. Resolução Conjunta SMA IBAMA/SP nº1 de 17 de fevereiro de 1994. DOE, n. 32, v. 104, seção 1, p. 47-48, Disponível em: < https://acervo.socioambiental.org/acervo/documentos/resolucao-conjunta-smaibamasp-1-de170294-define-vegetacao-primaria-e-secundaria> Acesso em: 21 jan. 2021.

Siminski A, Fantini AC. 2004. A classificação da Mata Atlântica do litoral catarinense em estádios sucessionais: ajustando a lei ao ecossistema. Floresta e Ambiente, 11(2):20-25, Disponível em: < http://s3.amazonaws.com/host-article-assets/ floram/588e2209e710ab87018b4613/fulltext.pdf > Acesso em: 05 abr. 2021.

Siminski A, Fantini AC, Reis MS. 2013. Classificação da vegetação secundária em estágios de regeneração da Mata Atlântica em Santa Catarina. Ciência Florestal, Cascavel, v. 23, n. 3, p. 369-378,. Disponível em: <http://cascavel.ufsm.br/ revistas/ojs-2.2.2/index.php/cienciaflorestal/article/view/10548/pdf>. Acesso em: 05 abr. 2021.

Sokal RR and Rohlf FJ. 2012. Biometry: the principles and practice of statistics in biological research. 4. ed. W.H. Freeman: San Francisco, USA.

SOS Mata Atlântica. Relatório Anual 2019, 2019. Disponível em: < https://www.sosma.org.br/wp-content/ uploads/2020/11/Relat\%C3\%B3rio-Anual-2019-SOS-Mata-Atl\%C3\%A2ntica.pdf >. Acesso em: 21 jan. 2021.

Tanus MR, Pastore M, Bianchini RS, Gomes EPC. 2012. Estrutura e composição de um trecho de Mata Atlântica no Parque Estadual das Fontes do Ipiranga, São Paulo, SP, Brasil. Hoehnea, 39(1):157-168. DOI: 10.1590/S2236-89062012000100010

van DYKE F. 2008. Conservation biology: foundations, concepts, applications. 2. ed. Dordrecht: Springer Netherlands.

Esta obra está licenciada com uma Licença Creative Commons Atribuição Não-Comercial 4.0 Internacional. 